\title{
SÍNTESE E CARACTERIZAÇÃO DE PENEIRAS MOLECULARES: SAPO-5
}

\author{
C. O. ROCHA ${ }^{1}$, P. S. B. NOBREGA ${ }^{2}$, C. M. B. M. BARBOSA ${ }^{3}$ e M.W. N. C. CARVALHO \\ 1,2,4 Universidade Federal de Campina Grande, Departamento de Engenharia Química \\ ${ }^{2}$ Universidade Federal de Pernambuco, Departamento de Engenharia Química \\ E-mail para contato: clariceoliveirarocha@gmail.com
}

\begin{abstract}
RESUMO - A redução do teor de enxofre na gasolina se apresenta como a melhor opção para se alcançar um ar e um meio ambiente mais limpos. Propostas têm aparecido visando à remoção desse elemento por intermédio dos processos de adsorção. Esse processo requer a utilização de adsorventes seletivos, com alta capacidade de adsorção do contaminante e regeneração dos mesmos. As peneiras moleculares vêm se mostrando como um excelente adsorvente, como é o caso do silicoaluminofosfato (SAPO). Elas apresentam grande área superficial específica, o que implica em uma estrutura altamente porosa. Assim, este trabalho tem como objetivo sintetizar SAPO-5, para obtenção de uma área superficial e diâmetro de poro maior, com adição de um reagente na síntese desse material. Para isso, foi realizada técnicas de caracterização, como DRX, EDX e BET. A área superficial teve um aumento de $19 \%$ na área BET, e no diâmetro do poro cerca de $63 \%$ com a adição do reagente para a obtenção de maior porosidade. Obteve-se um material altamente cristalino, com suas estruturas bastante ordenadas, com teores dos óxidos dos elementos característicos do SAPO-5.
\end{abstract}

\section{INTRODUÇÃO}

Segundo López et al. (2009), as zeólitas são microporosos aluminosilicatos cristalinos com importantes aplicações em catálise heterogênea, em processo de separação, adsorção e purificação. Elas são amplamente usadas no refino do petróleo e processos petroquímicos. O sistema microporoso zeólitas lhes confere importante propriedade de seletividade de forma e funcionalidade química. Em alguns casos, as propriedades catalíticas das zeólitas podem estar limitadas por problemas de transporte, devido às lentas difusões das reações e dos produtos desejados nos microporos $(0,3-1,5$ $\mathrm{nm})$. Outro problema principal é a desativação por bloqueio dos poros, causada pela formação de componentes pesados dentro dos microporos. Por outra parte, as peneiras moleculares mesoporosas, como MCM-41 (Mobil Composition of Matter No. 41), HMS (Hexagonal Mesoporous Sieve. IV) e SAPO-5, que foram sintetizadas a partir de 1990, e essa última nos últimos 10 anos, devido ao seu maior tamanho de poro, representaram um alto potencial para a conversão de moléculas volumosas. $\mathrm{O}$ interesse por esses sólidos vem crescendo e atualmente existe um número significativo de trabalhos que relatam a síntese, caracterização e aplicações catalíticas desses sólidos.

Zeólita do tipo microporosos vem atraindo estudos, principalmente, na área de adsorção e 


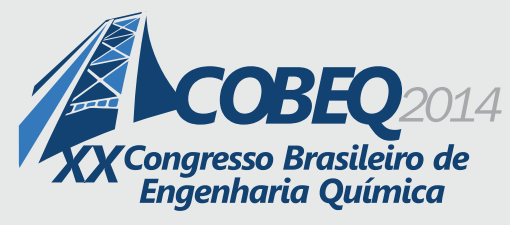

\section{9 a 22 de outubro de 2014 \\ Florianópolis/SC}

catálise. Tradicionalmente, a definição dos materiais do tipo zeólita foi restrita para materiais cristalinos constituídos de aluminosilicatos com $\mathrm{SiO}_{4}$ e $\mathrm{AlO}_{4}$ tetraedros. Em 1982, Wilson e colaboradores (apud. CHOI et al., 2006) desenvolveram uma nova classe com estruturas semelhantes as das zeólitas de cristais inorgânicos, denotado como $\mathrm{AlPO}_{4}-\mathrm{n}$ (n indica o tipo de estrutura), cujas estruturas são construídas com $\mathrm{AlO}_{4}$ e $\mathrm{PO}_{4}$. O curioso dos materiais é que parte do $\mathrm{Al}$ pode ser substituído com espécies metálicas diversas, incluindo $\mathrm{Si}, \mathrm{Mg}, \mathrm{Ti}, \mathrm{V}, \mathrm{Cr}, \mathrm{Mn}, \mathrm{Fe}$, Co, etc, o que permite a introdução de várias funções catalíticas. Por exemplo, Si, contendo aluminofosfato (SAPOs) possuindo sítios ácidos, tem sido extensivamente estudado como catalisadores para transformação do metanol em olefinas (CHOI et al., op. cit.).

O presente trabalho consiste em obter mais conhecimento sobre os aspectos relacionados à síntese, à caracterização e às aplicações catalíticas de peneiras moleculares micro-mesoporosas. Vários trabalhos vêm relatando o uso de compostos organosilano para obter a mesoporosidade, controlada em zeólitas. Determinados métodos foram utilizados por alguns autores, como Suzuki et al. (2008), que utilizaram o TPHAC - [3 - (trimetoxisilil) propil] hexadecildimetilamônio (a metodologia deste reagente está descrito no trabalho de HUTTINGER \& JUNG, 1989), como agente meso-estruturante para obter ZSM-5 mesoporoas, com poros entre 5 e $11 \mathrm{~nm}$ e a relação Si/Al entre 24 e 36. Esses autores realizaram um estudo comparativo da acidez e da atividade catalítica para o craqueamento do n-octano da zeólita ZSM-5 mesoporosa com ZSM-5 convencional, AlMCM-41 e uma sílica alumina amorfa. O ZSM-5 mesoporoso apresentou sítios de Brønsted fortes, como ZSM-5 convencional, mas em baixas concentrações, assim concluiu a formação parcial da estrutura zeólita nos materiais mesoporosos. Esses sólidos Al-MCM-41 e a sílica alumina amorfa mostraram uma acidez e atividade catalítica similar e menores do que a obtida com a ZSM-5.

Shanbhag et al. (2009) relataram a síntese de sodalita $(\mathrm{Si} / \mathrm{Al}=1)$ com uma estrutura micromesoporosa, usando o TPHAC como modelo. A síntese foi baseada em um tratamento hidrotérmico de géis, aquecidos a 100 e $150^{\circ} \mathrm{C}$ por 6 h. Foi possível obter uma sodalita mesoporosa com uma superfície específica 10 vezes maior e o volume poroso da sodalita microporosa 4 vezes maior. Esses sólidos mostraram boa atividade catalítica e regenerabilidade para reações de catálises básicas.

Recentemente, tem-se estudado a incorporação da mesoporosidade em SAPO-5, através de diferentes estratégias de síntese. $\mathrm{O}$ material com as melhores propriedades textuais e catalíticas em reações de alquilação catalítica ácida foi obtido, através da ciclohexilamina para manter a microporosidade e do modelo macio, contendo silício como o grupo cabeça o TPHAC para a geração dos mesoporos. A fim de otimizar o desempenho catalítico do material do modelo SAPO-5, foram realizadas sínteses, que sistematicamente variou-se a fonte de silício, o teor de silício e a quantidade do agente direcionador mesoporosos, na mistura da reação (DANILINA et al., 2011).

O SAPO-5 apresenta padrões característicos de difração de raios-X, nos quais podem ser utilizados, qualitativamente, para identificar o material e detectar a existência de outras formas cristalinas e, quantitativamente, para determinar o grau de pureza e/ou a cristalinidade (GIANNETTO, 1989).

Neste trabalho, será utilizado como padrão o difratograma de raios-X, característico da estrutura 
AFI, que está apresentada na Figura 1, indicando os índices de Miller dos planos cristalográficos da sua estrutura.

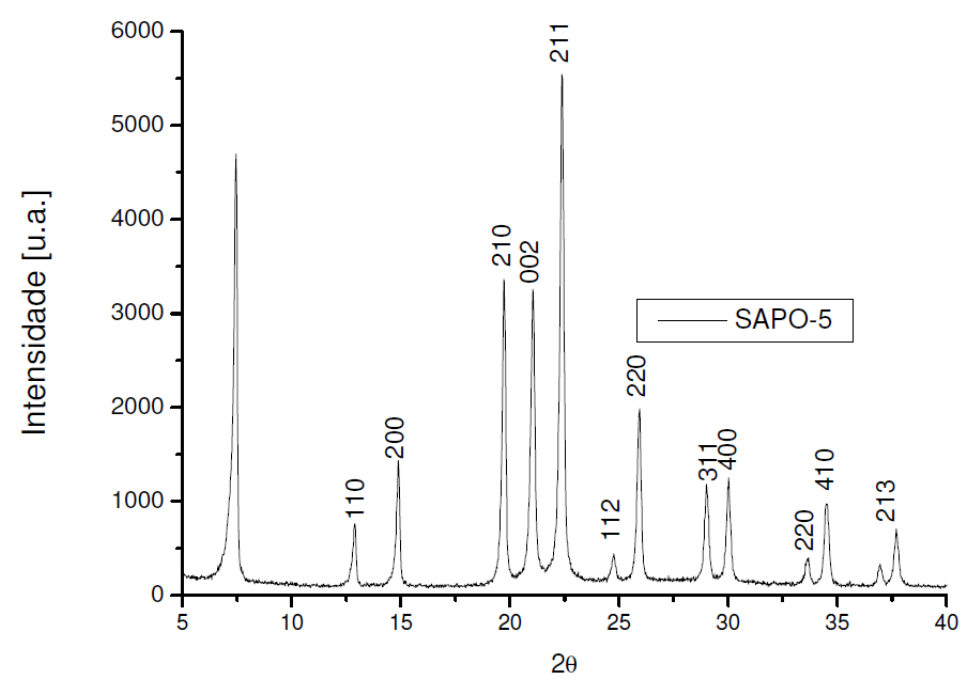

Figuras 1 - Curvas de difração de raios-X da amostra padrão do SAPO-5

FONTE: IZA-SC (http://topaz.ethz.ch/IZA-SC/PDFfiles.htm)

Danilina et al. (2010) sintetizaram o silicioaluminofosfato (SAPO) cristalino do tipo AFI, com alto teor de silício hidrotermicamente, usando um modelo contendo silício, como grupo no topo e ciclohexilamina, para manter a microporosidade. As propriedades estruturais e catalíticas do novo modelo foram amplamente caracterizadas e comparadas com os de microporosos SAPO-5 e HZSM-5. A síntese do H-SAPO-5M (mesoporosos) foi obtida utilizando TPHAC como direcionador. Outros procedimentos foram utilizados para obter SAPO mesoporosos, como o modelo com carbono preto, surfactantes anfifílicos, com moléculas de fósforo e alumínio. Os resultados para esses modelos não foram de um material mesoporoso, foram amorfa, com impuras e pouco ácidas. H-SAPO-5M mostrou um grande número de sítios ácidos de Brønsted e alta reatividade, semelhantes aos de microporosos H-SAPO-5, na dessorção à temperatura programada do n-propilamina, na isomerização do 2-metil-2penteno e no craqueamento monomolecular do propano. H-SAPO-5M indica que as reações ocorreram no domínio mesoporosos, de acordo com a grande área de superfície externa.

Com isso, este trabalho tem como objetivos sintetizar e caracterizar as peneiras moleculares SAPO-5 para a obtenção de um material com área superficial e diâmetro de poro maior. Assim, apresenta-se uma maior área superficial externa, oferecendo mais espaço para as moléculas volumosas se difundirem e reagirem. Foi utilizado o SAPO-5, por ser um material econômico para a realização de sua síntese.

\section{EXPERIMENTAL}

\subsection{SAPO-5 Microporoso}


A síntese do SAPO-5 foi realizada usando o meio bifásico, baseado na metodologia de Urbina (1997) e adaptada por Cabral (2008). Foram utilizados os seguintes reagentes na preparação das amostras do SAPO-5: Tetraetil Ortossilicato -TEOS (98\%, Aldrich), pseudobohemita (cedida pelo CENPES/PETROBRAS), trietilamina (Merck), ácido fosfórico (85\%, Merck), n-hexanol (Merck) e brometo de hexadeciltrimetilamônio - CTMABr (Merck).

\subsection{SAPO-5 Microporosidade maior}

O procedimento de síntese é análogo ao do SAPO-5 microporoso, de acordo com a metodologia utilizada por Danilina et al. (2010). Esses autores utilizaram o reagente TPHAC como um modelo para gerar a mesoporosidade. Kim, Choi e Ryoo (2010) comprovaram que o reagente TPHAC pode ser substituído por 3 - [(trimetoxissilil) propil]-cloreto de octadecil dimetilamônio (TPOD) (SigmaAldrich), possui as mesmas características químicas do TPHAC e, por ser um reagente pronto, não precisa sintetizá-lo, ou seja, diminuindo a erros experimentais. Com isso, para esse trabalho foi utilizado o reagente TPOD para aumentar a porosidade do material.

\subsection{Caracterização}

Para o ensaio de difração de raios-X, foi utilizado um equipamento da marca Shimadzu, modelo XRD 600 com fonte de radiação $\mathrm{CuK} \alpha$, com varredura de $2 \theta=5$ a $40^{\circ}$. Para o de Espectroscopia de Raios-X por energia dispersiva (EDX), o equipamento utilizado foi da marca Shimadzu, modelo EDX - 720 Energy dispersive X- Ray spectrometer. A tensão de operação foi de $15 \mathrm{KV}$ e a corrente de $100 \mu \mathrm{A}$. Para a realização da Caracterização Textural por BET, as amostras foram submetidas a um pré-tratamento a $350^{\circ} \mathrm{C}$ sob fluxo de nitrogênio por 10 horas para remoção de umidade e impurezas. Foi utilizado um equipamento da Micromeritics modelo ASAP2020 (CABRAL, 2008).

\section{RESULTADOS E DISCUSSÕES}

As curvas de difração de raios-X do SAPO-5 são caracterizados por apresentarem picos de reflexão, na faixa de $2 \theta$ compreendidos entre 5 e 45 graus, como foi apresentado na Figura 1. Diante das Figuras 2 e 3, comprova-se que as fases do SAPO-5 foram alcançadas, por apresentarem estruturas ordenadas e cristalinas, livre de fase amorfa. 


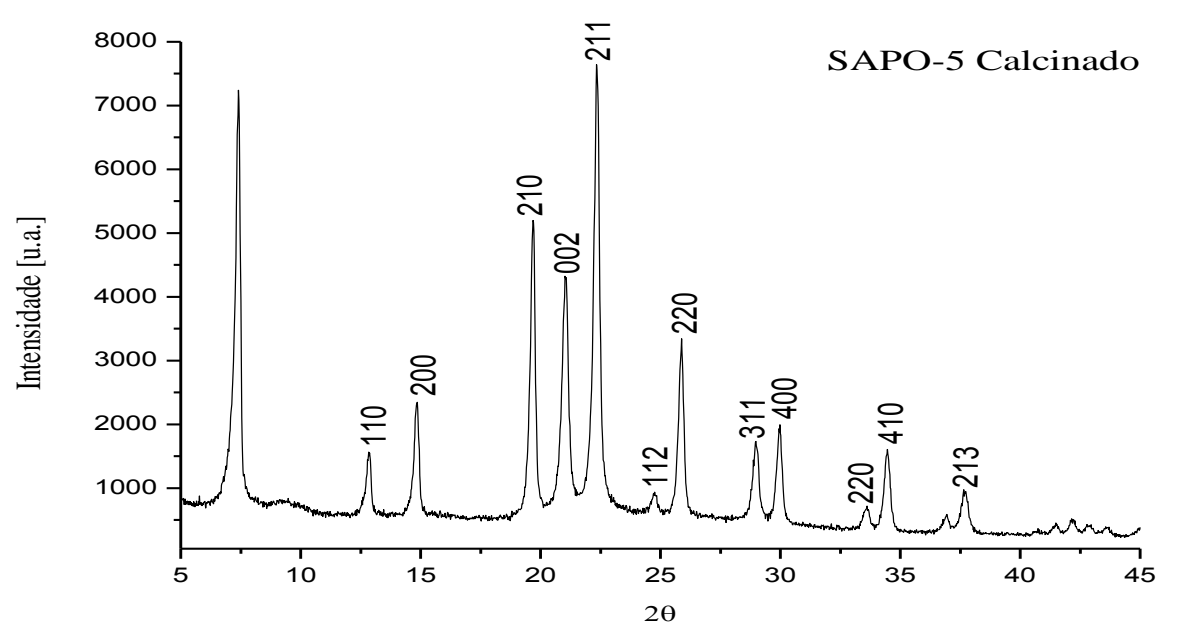

Figura 2 - Curvas de difração de raios-X para o suporte SAPO-5

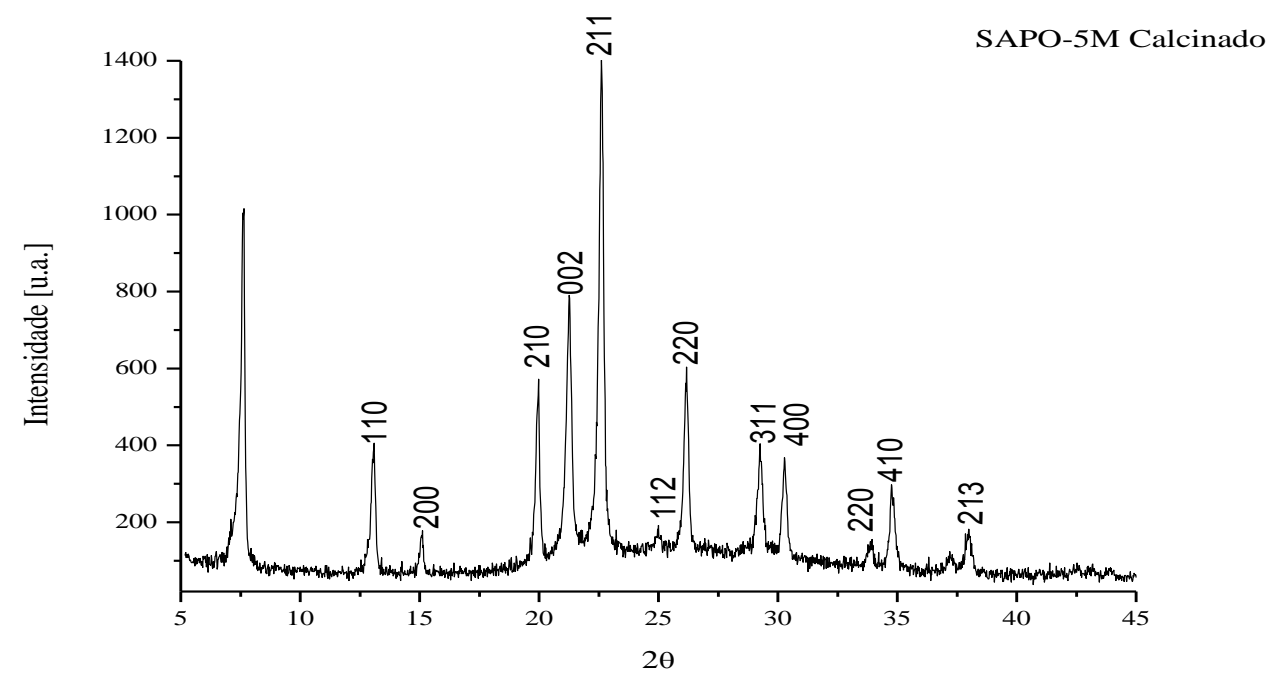

Figura 3 - Curvas de difração de raios-X para o suporte SAPO-5 com Microporosidade maior.

Nas Figuras 2 e 3, confirma-se que a síntese foi efetiva para o suporte e verifica-se que a calcinação não comprometeu a estrutura dos poros delimitados por anéis de 12 tetraedros. É interessante notar, na Figura 3, que a intensidade de difração relativo dos planos do calcinado e do não calcinado foi praticamente a mesma, com intensidade cerca de 1400 (221), indicando que a adição do reagente para uma porosidade maior não afetou na cristalinidade do suporte. Esse resultado está de acordo com os resultados obtidos anteriores, por Danilina et al. (2011), Danilina et al. (2010), Utchariyajit e Wongkasemjit (2010). 
Os resultados da análise de EDX (Espectroscopia de raios-X por Energia Dispersiva) estão representados na Tabela 1:

Tabela 1: Análise semi-quantitativa dos suportes (SAPO-5 e SAPO-5M)

\begin{tabular}{c|c|c|c} 
Amostras & $\mathrm{SiO}_{2}(\%)$ & $\mathrm{Al}_{2} \mathrm{O}_{3}(\%)$ & $\mathrm{P}_{2} \mathrm{O}_{5}(\%)$ \\
\hline SAPO-5 & 27,44 & 46,08 & 26,45 \\
\hline SAPO-5M & 38,69 & 34,70 & 26,61
\end{tabular}

A Tabela 1 confirma a presença dos elementos característicos do suporte SAPO-5. Leite, Carvalho e Pereira (2010) confirmam que o método utilizado na preparação do suporte foi eficiente.

A Tabela 2, abaixo, mostra os resultados da adsorção de nitrogênio a 77,350 K, a partir do método BET, dos suportes SAPO-5. A partir dessas análises, foram obtidas as áreas superficiais e os diâmetros dos poros.

Tabela 2 - Dados da Área Superficial e Diâmetro de Poro determinados pelo método de BET

\begin{tabular}{c|c|c} 
Amostras & BET $\left(\mathrm{m}^{2} / \mathrm{g}\right)$ & Diâmetro do poro $(\AA)$ \\
\hline SAPO-5 & 185,4 & 7,011 \\
\hline SAPO-5M & 229,8 & 19,197
\end{tabular}

A adição do reagente TPOD fez com que a área BET do SAPO-5 microporoso aumentasse 19\% de sua área, comparado com o SAPO-5M. Isso também pode ser visto no trabalho de Danilina, et al. (2010), que aumentou cerca de $11 \%$ de sua área. Assim, este trabalho conseguiu alcançar o objetivo, já que aumentou consideravelmente a área e o diâmetro.

Já em relação ao diâmetro do poro, ele teve um aumento de aproximadamente 63\% do SAPO-5 para o SAPO-5M, enquanto Danilina, et al. (2010) e Danilina, et al. (2011) conseguiram aumentar cerca de $83 \%$. Vale destacar que esses aumentos nas áreas BET e nos diâmetros dos poros são interessante para o processo de adsorção, pois quanto maiores forem, mais moléculas volumosas podem ser adsorvidas, principalmente, os compostos sulfurados encontrados na gasolina, como por exemplo, os tiofenos.

Portanto, a síntese do SAPO-5 microporoso foi realizada com total êxito. Formou-se um material altamente cristalino, livre de fase amorfa e obteve-se a fase AFI pretendida, com área superficial, volume de poro e diâmetro de poro dentro do esperado - com o aumento de $19 \%$ na sua área - comprovando que o reagente TPOD pode ser utilizado com esse objetivo. Isso é importante, 


\section{9 a 22 de outubro de 2014 \\ Florianópolis/SC}

pois para a utilização desse material como adsorvente quanto maior for sua área e seu diâmetro mais moléculas volumosas, com algum tipo de contaminante, ele poderá adsorver. Com este trabalho ficou comprovado que a utilização desse adsorvente - SAPO-5M - pode ser utilizado em alguns processos de retirada de contaminantes que degradam o ambiente bem como o auto custo nos processos utilizando o hidrogênio, a exemplo do processo de dessulfurização, para diminuir a quantidade de enxofre em combustíveis, (cf. ROCHA, no prelo), pois, além desse processo ter custo baixo, o adsorvente, também, é econômico na sua síntese.

\section{REFERÊNCIAS}

CABRAL, R. P. B. Síntese e Avaliação de NiMo/BETA e NiMo/SAPO-5 no Hidrocraqueamento do Cumeno com Piridina. Tese de Doutorado. Universidade Federal de Campina Grande-UFCG, 2008.

CHOI, M., SRIVASTAVA, R., RYOO, R. Orga-nosilane surfactant-directed synthesis of mesoporous aluminophosphates constructed with crystalline microporous frameworks. Chem. Commun., 2006, p. 4380-4382.

DANILINA, N., KRUMEICH, F., BOKHOVEN, J. A. van. Hierarchical SAPO-5 catalysts active in acid-catalyzed reactions. Journal of Catalysis 272, 2010, p. 37-43.

DANILINA, N., CASTELANELli, S. A., TROUS-SARD, E., BOKHOVEN, J. V. Influence of synthesis parameters on the catalytic activity of hierarchcal SAPO-5 in space-demanding alkylation reactions. CatalysisToday, 2011, p. xxx-xxx.

GIANNETTO, G. P. Zeolitas, Catacteristicas, Propiedades y Aplicaciones Industriales. Edit-Editorial Innovación Tecnológica, 1989.

HUTTINGER, K.J. \& JUNG, M.F., Kinetik der Synthese von Trialkyl[-3-(trimethoxysilyl)-propyl]ammoniumchloriden und deren antimikrobielle Wirkung als fixierte Biozide. Chem. Ing. Tech. 61, 1989, p. 258-259.

IZA-SC $\quad-\quad$ IZA Structure Commission. Disponível em: <http://topaz.ethz.ch/IZASC/Atlas_pdf/AFI.pdf>. Acesso em: 05 Maio. 2013.

KIM, J.; CHOI, M.; RYOO, R. Effect of mesoporosity against the deactivation of MFI zeolite catalyst during the methanol-to-hydrocarbon conversion process. Journal of Catalysis 269, 2010, p. 219-228.

LEITE, C. E. T.; CARVAlHO, M. W. N. C.; PEREIRA, K. R. O. Síntese e Caracterização de Suportes Catalíticos do tipo Silicoaluminofosfático. $19^{\circ}$ Congresso Brasileiro de Engenharia e Ciência dos Materiais - CBECiMat, Campos do Jordão, SP, Brasil, 2010, p. 2386-2393.

LÓPEZ, C. M.; SAZO, V.; URBINA, C.; GARCÍA, M. G. Tamices moleculares que combinan micro y meso porosidad: una revisión. Avances em Química, 4(3), 2009, p. 113-125.

ROCHA, C. O. (No prelo) Remoção de Enxofre na mistura Heptano-Tiofeno por meio do Processo de Adsorção sobre Zn/SAPO-5 e Ni/SAPO-5. Tese em Doutoramento pela Universidade Federal de Campina Grande.

SHANBHAG, G.V., CHOI, M, KIM, J., RYOO, R. Mesoporous sodalite: A novel, stable solid catalyst for base-catalyzed organic transformations. Journal of Catalysis., 264, 2009, p. 88-92. 
SUZUKI, K., AOYAGI, Y., KATADA, N., CHOI, M., RYOO, R., NIWA, M. Acidity and catalytic activity of mesoporous ZSM-5 in comparison with zeolite ZSM-5, AL-MCM-41 and silica-alumina. CatalysisToday, 132, 2008, p.38-45.

URBINA, M. M. Síntese do Silico-Alumino-Fosfato SAPO-5 em Meio Aquoso e Bifásico, sua Caracterização e Avaliação Catalítica. Tese de Doutorado - Universidade Federal de São CarlosUFSCar, São Carlos, 1997.

UTCHARIYAJIT, K.; WONGKASEMJIT, S. Effect of synthesis parameters on mesoporous SAPO-5 with AFI-type formation via microwave radiation using alumatrane and silatrane precursors. Microporous and Mesoporous Materials 135, 2010, p. 116-123. 